\title{
Epidemiological characteristics of breakthrough varicella infection during varicella outbreaks in Shanghai, 2008-2014
}

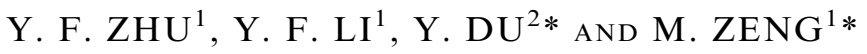 \\ ${ }^{1}$ Department of Infectious Diseases, Children's Hospital of Fudan University, No. 399 Wanyuan Road, \\ Shanghai, China \\ ${ }^{2}$ Department of Immunization Program, Minhang District Center for Disease Control and Prevention, \\ Shanghai, China
}

Received 7 October 2016; Final revision 20 February 2017; Accepted 18 March 2017; first published online 27 April 2017

\section{SUMMARY}

The outbreaks of varicella occurring in kindergartens and schools are increasingly notified in Shanghai despite the implementation of one-dose varicella vaccination. We analyzed surveillance data on the notified outbreaks of varicella in Minhang District of Shanghai during 2008-2014. A total of 13511 varicella cases and 154 outbreaks involving $1558(11 \cdot 5 \%)$ cases were reported. Annual attack rates of outbreak-associated varicella in outbreak classes were $5 \cdot 5 \%-12 \%$. The mean age of the outbreak-associated cases was 8.6 $13 \cdot 1$ years. Among 1558 outbreak cases, 660 $(42 \cdot 4 \%)$ received one-dose varicella vaccine previously. The proportions of breakthrough varicella infection during outbreaks ranged from $21 \cdot 5 \%$ in 2008 to $86.1 \%$ in 2014 . Annual breakthrough infection rates in outbreak classes ranged from $5 \cdot 4 \%$ to $7 \cdot 4 \%$. Breakthrough cases as index cases results in $9.7 \%$ of outbreaks, and the average duration of outbreaks was significantly longer in vaccinated cases as index cases than in unvaccinated cases as index cases $(11 \cdot 3 \pm 5 \cdot 8$ days $v s$. $8 \cdot 6 \pm 6 \cdot 1$ days, $P<0 \cdot 05$ ). The mean time of breakthrough infection since vaccination was $6 \cdot 2 \pm 2 \cdot 3$ years (range $0 \cdot 6-13 \cdot 4$ years). One-dose varicella vaccination cannot prevent the varicella outbreaks in kindergartens and schools. A second dose of varicella vaccine should be recommended for children.

Key words: Breakthrough infection, outbreak, vaccination, varicella.

\section{INTRODUCTION}

Varicella is usually a highly contagious disease in childhood caused by primary varicella-zoster virus (VZV). The transmission rate is high and secondary attack rates are between $70 \%$ and $90 \%$ among susceptible siblings within a household and $12-33 \%$ in

\footnotetext{
* Author for correspondence: Dr M. Zeng and Y. Du, Department of Infectious Diseases, Children's Hospital of Fudan University, 399 Wanyuan Road, Shanghai 201102, China and Department of Immunization Program, Minhang District Center for Disease Control and Prevention, Shanghai, China. (Email: zengmeigao@) aliyun.com,amisydu@163.com)
}

school classrooms before the vaccine era [1]. Typically, the virus is introduced into the susceptible school-aged or preschool children and results in varicella outbreaks in schools and childcare centers, which had a serious impact on the normal school education.

Live attenuated Oka strain varicella vaccine was recommended for vaccination among healthy preschool children as a single dose in Shanghai since 1997 if a child's parent is willing to pay for it. Published data showed that the effectiveness of onedose varicella vaccine is $75-94 \%$ [2-8], and the implementation of one-dose varicella vaccination results in 
a substantial reduction in morbidity and mortality of varicella $[9,10]$. Although one-dose varicella vaccine is highly effective in containing natural varicella cases, vaccine effectiveness decreases with time along with evidence of waning immunity [11]. Breakthrough varicella infections were reported after one-dose of varicella vaccination and the attack rates of breakthrough cases in vaccinated children were about $1 \cdot 6-6 \%$ due to both primary vaccine failure and waning vaccine-induced immunity [2, 3, 11, 12]. The 1999-2005 surveillance data on varicella vaccination in Shanghainese children showed that the incidence of varicella in vaccinated children was 4.35 time lower than that in unvaccinated children [13]. Varicella outbreaks and breakthrough varicella infections have been reported increasingly in Shanghai in recent years despite the fact that the coverage rate of one-dose varicella vaccination was estimated to reach about $85-95 \%$ among children attending kindergarten and primary school. This situation challenged the current one-dose vaccination policy. The aim of this study was to understand the epidemiological characteristics of varicella outbreaks and outbreak-associated breakthrough infections in Shanghai and provide evidence for effective prevention and control of varicella.

\section{METHODS}

\section{Varicella surveillance}

Mandatory reporting of varicella in Shanghai began in 2006 after varicella vaccine was more widely used in children. Active surveillance of varicella outbreaks was enhanced in Minhang District of Shanghai since 2008. Local health providers and physicians are required to notify varicella cases directly to the Shanghai Municipal Center for Disease Control and Prevention (CDC) within $24 \mathrm{~h}$ via internet-based surveillance system. The epidemiologic information is recorded for a clinically diagnosed varicella case: age, sex, current home address, household registration, symptoms onset date, diagnosis date, hospitalization date. Kindergartens, schools and universities obligatorily report the varicella outbreaks to the public health agency. Meanwhile, the district CDC actively monitors the varicella outbreaks in institutions via internet reporting system. In response to varicella outbreaks, public health staffs conduct the field epidemiological investigation and take the control measures. Since 2013, active post-exposure active immunization was recommended for exposed healthy children in outbreak classes and schools, including those who had no clear varicella history and who had a single dose of varicella vaccination at least 5 years ago.

\section{Definition}

Varicella was diagnosed based on clinical manifestations with an acute maculopapulovesicular rash appearing on the scalp, face, and trunk without other apparent cause in a child or an adult; the typical rash usually evolves from erythematous macules to fluid-filled vesicles and crusting characteristic of umbilication. However, breakthrough varicella is usually mild and characteristic of fewer lesions and atypical vesicles, but typical appearance on the scalp, face, and trunk. Varicella was clinically diagnosed by infectious disease specialists.

An outbreak was defined as five or more new cases of varicella occurring within 21 days (the longest incubation period of varicella) in an institution (school or kindergarten or university). If 10 or more cases of varicella occurred within 1 week in an institution, such a situation was defined as a public health emergency. The duration of an outbreak was defined as a period from the date of varicella onset in the index case to the date of varicella onset in the last case. Breakthrough varicella infection is defined as any VZV infection occurs $>42$ days after the varicella vaccination [4]. Index case was defined as the first varicella case in an outbreak.

The annual incidence rate of varicella was defined as the number of reported varicella cases divided by the number of the general population of inhabitants. The annual attack rate of outbreak-associated varicella in classes was defined as the number of outbreak-associated varicella cases divided by the total number of children and university students in outbreak classes. The annual attack rate of outbreak-associated varicella in schools was defined as the number of outbreak-associated varicella cases divided by the total number of children and university students in outbreak schools.

Breakthrough infection rate was defined as the proportion of breakthrough infection cases among individuals who had received varicella vaccination in outbreak classes.

The cumulative proportion of population vaccinated was defined as the number of age-specific population vaccinated divided by the total number of the corresponding age-specific population by the end of 2014, based on the 2013-2014 Immunization Information Registry System and Census of Minhang District. 


\section{Data collection}

The demographic information of varicella case was obtained from Shanghai notifiable infectious disease reporting system. The varicella vaccination status, vaccine batch lot and vaccine manufacturer for outbreak-associated cases and their exposed classmates were obtained through Immunization Handbook (2008-2014) and Shanghai's Immunization Information Registry System developed since 2013. During 20082014, two varicella vaccines were used for children in Shanghai, including the imported varicella vaccine $\left(\right.$ Varilrix $^{\circledR}$ ) produced by GlaxoSmithKline and the domestic varicella vaccine produced by the Shanghai Institute of Biological Products, Shanghai. The domestic vaccine (Shanghai) introduced the Oka strain VZV and contains similar concentrations of Oka strain VZV with $>2000$ plaque forming unit/dose (0.5 ml).

During 2012-2014, 176 vaccinated children and 214 unvaccinated children in outbreak classes were followed-up to observe the severity of illness.

The data collection was a part of routine varicella surveillance program and exempt from ethical approval after the review of Ethics Committee of Shanghai Municipal CDC.

\section{Statistical analysis}

Data were double entered using Epidata $3 \cdot 1$ and comparisons in between groups were performed using SPSS statistical software, version 13.0 (SPSS Inc., USA). The $\chi^{2}$ test or Fisher's test was used to compare proportions between groups, as appropriate. Continuous variables were analyzed by Student's $t$ test. A $P$ value $<0.05$ was considered significant.

\section{RESULTS}

\section{Varicella cases and outbreaks}

From 2008 to 2014, a total of 13511 varicella cases were reported in Minhang District of Shanghai, and the annual incidence of varicella ranged from $66.8 /$ 100000 to $133 \cdot 4 / 100000$ with an average annual incidence of $91 \cdot 1 / 100000$ among the local population (Table 1). The mean age of 13511 varicella cases was $12 \cdot 9 \pm 9 \cdot 5$ years $(95 \%$ CI $12 \cdot 7-13 \cdot 1$ years, range 1 day to 84.9 years); male cases were $8334(61 \cdot 7 \%)$ and female cases were 5177 (38.3\%).

During a period of 7 years, 154 varicella outbreaks (including one public health emergency in a private primary school in 2011) and $1558(11 \cdot 5 \%)$ outbrea$\mathrm{k}$-associated cases were reported. The attack rates of varicella in outbreak-associated classes ranged from $12.0 \%$ in 2009 to $5 \cdot 5 \%$ in 2014 and declined significantly in 2014 (Table 1). The attack rates in outbreak-associated schools and kindergartens ranged from $0.6 \%$ in 2008 to $1.4 \%$ in 2014 (Table 1). The 154 varicella outbreaks occurred in kindergartens and schools, including $84(54.5 \%)$ outbreaks involving $898(57 \cdot 6 \%)$ cases in primary schools, $30(19 \cdot 5 \%)$ outbreaks involving $245(15.7 \%)$ cases in middle schools, $29(18 \cdot 8 \%)$ outbreaks involving $228(14 \cdot 6 \%)$ cases in kindergartens, and $11(7 \cdot 1 \%)$ outbreaks involving $187(12 \cdot 0 \%)$ cases in universities.

As shown in Fig. 1, occurrence of varicella in Shanghai has two peak seasons (from March to May and from October to December soon after school opening) and two off-seasons (from January to February and from July to August on school vacation) in both vaccinated group and unvaccinated group.

\section{Characteristics of breakthrough varicella during outbreaks}

The mean age of 1558 outbreak-associated varicella cases was $8 \cdot 6 \pm 3 \cdot 1$ years $(95 \%$ CI $8 \cdot 3-8.8$ years, range $3.0-31.8$ years); male were $960(61.6 \%)$ and female were $598(38 \cdot 4 \%)$. Compared with the overall cases, the outbreak-associated varicella cases were significantly younger $(P<0 \cdot 05)$ (Table 2$)$.

Among 1558 outbreak-associated cases, 660 (42.4\%) were breakthrough cases who had received one dose of varicella vaccine at age $>1$ year and $898(57 \cdot 6 \%)$ were unvaccinated. The ratios of male-female were 1.5:1 (399:261) for vaccinated cases and 1.7:1 (560:338) for unvaccinated cases, respectively $(P>0 \cdot 05)$. The mean ages were $11.9 \pm 5.7$ years $(95 \%$ CI $10.5-13.3$ years, range $3 \cdot 0-17 \cdot 7$ years) for vaccinated cases and $8 \cdot 2 \pm$ $2 \cdot 5$ years $(95 \%$ CI $8 \cdot 0-8 \cdot 4$ years, range $3 \cdot 4-31 \cdot 8$ years) for unvaccinated cases, respectively $(P<0 \cdot 05)$ (Table 2). As shown in Table 3, 654 (99.1\%) of 660 breakthrough cases were aged 3-14 years, among whom the proportion of accumulative vaccination reached around $90 \%$; only six cases $(0 \cdot 01 \%)$ were aged $15-17 \cdot 7$ years, among whom the proportion of accumulative vaccination reached $54 \cdot 2 \%$.

The proportions of breakthrough varicella showed an increasing trend with the year from $21 \cdot 5 \%(79 / 368)$ in 2008 to $86 \cdot 1 \%(204 / 237)$ in 2014 ; the breakthrough infection rates in outbreak classes were $7 \cdot 4 \%(64 / 861)$ 
Table 1. Annual attack rates of outbreak-associated varicella in schools and kindergartens and annual incidence of varicella in Minhang District during 2008-2014

\begin{tabular}{|c|c|c|c|c|c|c|}
\hline \multicolumn{4}{|c|}{ Community setting } & \multicolumn{3}{|c|}{ Outbreak setting } \\
\hline Year & $\begin{array}{l}\text { Number of } \\
\text { cases }\end{array}$ & $\begin{array}{l}\text { Entire } \\
\text { population }\end{array}$ & $\begin{array}{l}\text { Incidence } \\
(1 / 100000)\end{array}$ & $\begin{array}{l}\text { Number of } \\
\text { cases }\end{array}$ & $\begin{array}{l}\text { Number of students in } \\
\text { outbreak classes } \\
\text { (Attack rate \%) }\end{array}$ & $\begin{array}{l}\text { Number of students in } \\
\text { outbreak schools and } \\
\text { kindergartens (Attack rate \%) }\end{array}$ \\
\hline 2008 & 1233 & 1845742 & $66 \cdot 8$ & 368 & $4416(8 \cdot 3)$ & $60852(0 \cdot 6)$ \\
\hline 2009 & 1752 & 1845832 & $94 \cdot 9$ & 200 & $1663(12 \cdot 0)$ & $27051(0 \cdot 7)$ \\
\hline 2010 & 2251 & 1861558 & $120 \cdot 9$ & 233 & $2227(10 \cdot 5)$ & $24288(1 \cdot 0)$ \\
\hline 2011 & 2614 & 1961607 & $133 \cdot 3$ & 281 & $2406(11 \cdot 7)$ & $20793(1 \cdot 4)$ \\
\hline 2012 & 2118 & 2429376 & $87 \cdot 2$ & 143 & $1266(11 \cdot 3)$ & $12469(1 \cdot 1)$ \\
\hline 2013 & 1466 & 2429376 & $60 \cdot 3$ & 96 & $1119(8 \cdot 6)$ & $10587(0 \cdot 9)$ \\
\hline 2014 & 2077 & 2452750 & $84 \cdot 7$ & 237 & $4293(5 \cdot 5)$ & $16405(1 \cdot 4)$ \\
\hline Total & 13511 & 14826241 & $91 \cdot 1$ & 1558 & $17390(9 \cdot 0)$ & $156040(1 \cdot 0)$ \\
\hline
\end{tabular}

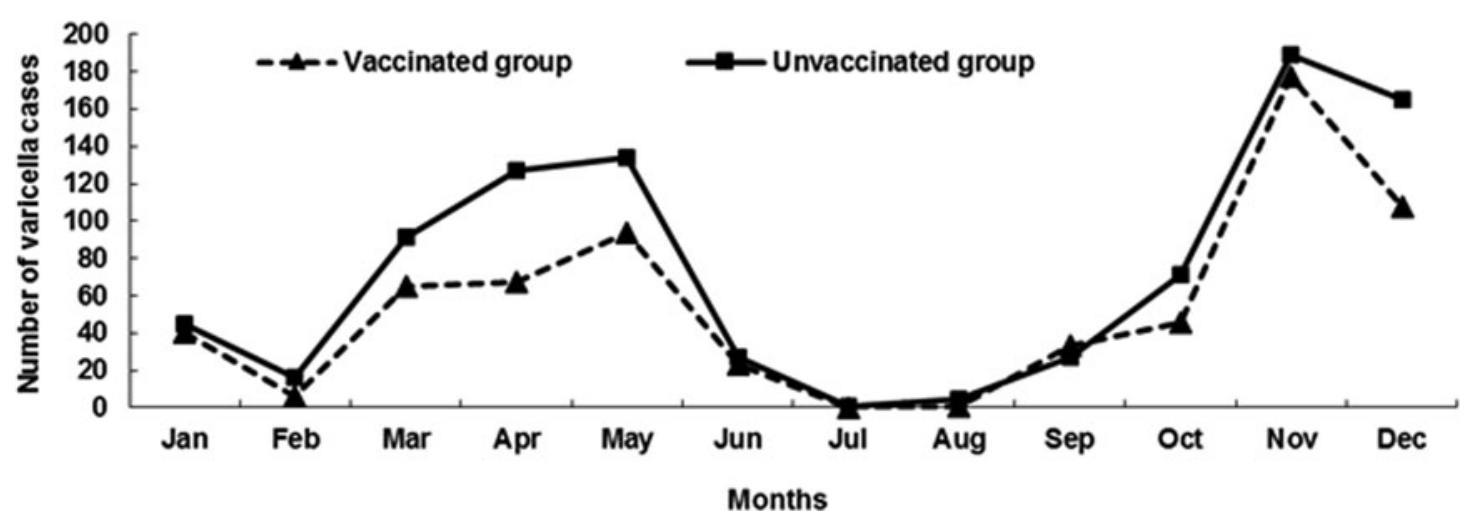

Fig. 1. Accumulated monthly number of outbreak-associated varicella cases among vaccinated and unvaccinated children in Minhang District during 2008-2014.

in 2013 and $5 \cdot 4 \%(204 / 3753)$ in 2014 , respectively (Table 4). In 15 (9.7\%) of 154 outbreaks, the index case was a breakthrough varicella case.

The average duration of outbreaks was $9 \cdot 0 \pm 6 \cdot 1$ days (range 1-19 days). The average duration of outbreaks was $11.3 \pm 5.8$ days (range $1-18$ days) in vaccinated breakthrough cases as index cases and $8 \cdot 6 \pm 6 \cdot 1$ days (range 1-19 days) in unvaccinated cases as index cases, with significant difference between the two groups $(P<0 \cdot 05)$.

The mean interval between vaccination and breakthrough infection was $6 \cdot 2 \pm 2 \cdot 3$ years $(95 \%$ CI $6 \cdot 1-$ $6 \cdot 6$ years, range $0.6-13 \cdot 4$ years). Of 660 breakthrough cases, $56(8 \cdot 5 \%)$ developed breakthrough infection $<3$ years after vaccination and $604(91 \cdot 5 \%)$ developed breakthrough infection $\geqslant 3$ years after vaccination; the number of breakthrough cases increased with the time since vaccination within 7 years after vaccination (Table 5).
Among 660 breakthrough cases, 445 (67.4\%) received a single dose of domestic varicella vaccine, $112(17 \%)$ received a single dose of imported varicella vaccine and the remaining $103(15.6 \%)$ could not obtain the detailed record of vaccine manufacturer. The average interval of breakthrough since vaccination were $6 \cdot 3 \pm 2 \cdot 2$ years $(95 \%$ CI $6 \cdot 1-6 \cdot 6$ years, range $3.9-12.9$ years) for domestic vaccine and $7 \cdot 8 \pm$ $12 \cdot 8$ years $(95 \%$ CI $4 \cdot 7-11$ years, range $3 \cdot 0-13 \cdot 4$ years) for imported vaccine, respectively, with no statistical difference $(P>0 \cdot 05)$.

On the basis of clinical observation, fever (oral temperature $\left.>37 \cdot 5^{\circ} \mathrm{C}\right)$ was observed in $46(26.1 \%)$ of 176 vaccinated varicella children and $117(54 \cdot 7 \%)$ of 214 unvaccinated varicella children $(P>0.05)$; numbers of lesions $>50$ was observed in $14(8.0 \%)$ of 176 vaccinated varicella children and $109(50.9 \%)$ of 214 unvaccinated varicella children $(P>0 \cdot 05)$. No severe complication was observed. 


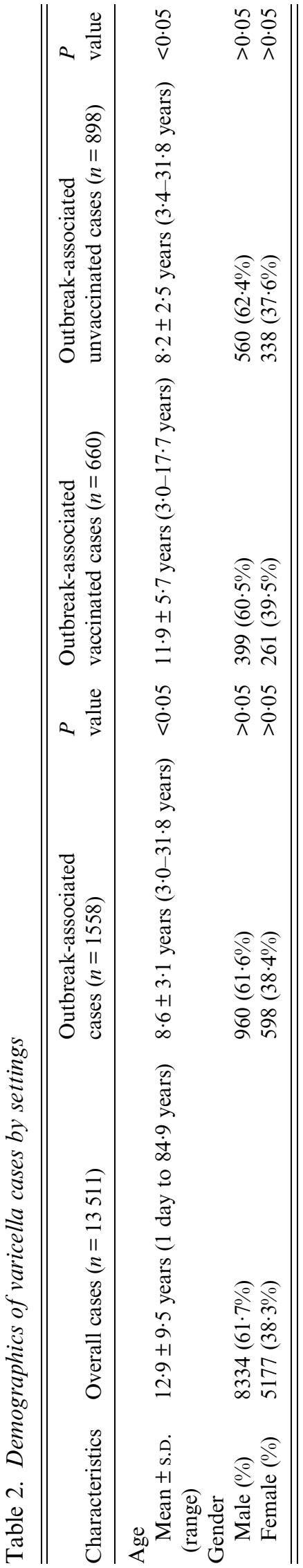

\section{DISCUSSION}

Varicella outbreaks in schools and kindergartens have gained attention from the public health and schools in Shanghai. This study showed that breakthrough varicella infection accounted for $42.4 \%$ of outbreak-associated cases with an increasing trend over year during 2008 through 2014 in Minhang District of Shanghai. A surveillance study conducted in Fengtai District of Beijing during 2008-2012 showed that $61 \%$ of outbreak-associated varicella cases were vaccinated previously [12]. These epidemiological studies raise our awareness of varicella breakthrough infection after one-dose childhood varicella vaccination and highlight that one-dose varicella vaccine does not prevent outbreaks in kindergartens and schools even in highly vaccinated school children. Live varicella attenuated vaccine was introduced in Shanghai in 1997 and a retrospective surveillance study conducted between 1999 and 2005 in Shanghai showed that one-dose varicella vaccine effectiveness is $77 \%$ [13]. This study provides an additional strong evidence for public health decision-makers to recommend universal two-dose childhood varicella vaccination program in Shanghai and regions where one-dose varicella vaccination is recommended.

Although this study observed a high varicella vaccination rate up to $90 \%$ among children aged 3-14 years old, varicella outbreaks occurred frequently in kindergarten and school settings. A study from the USA observed that $99 \%$ vaccination coverage of onedose varicella vaccine was not sufficient to prevent the outbreak in an elementary school and most of outbreak-associated cases were vaccinated students [4]. A higher degree of effectiveness of varicella vaccination is needed in order to interrupt transmission and to prevent outbreaks in settings with high contact rates. We observed that the peak seasons of natural and breakthrough varicella appeared 2 months after school opening. Thus, children should be vaccinated against varicella vaccine prior to kindergarten and school entry.

Breakthrough varicella infection may affect the public confidence in vaccine. The breakthrough infection rates in outbreak classes were $5 \cdot 4-7 \cdot 4 \%$, higher than the rates in community setting in Taiwan $(0 \cdot 7-$ $2 \cdot 5 \%)$ and Beijing $(0 \cdot 12-2 \cdot 14 \%)[2,12]$. The difficulty in early diagnosis and recognition of mild and atypical contagious breakthrough varicella will result in effective transmission, secondary outbreak and delayed response to the outbreaks. In fact, we observe much milder symptoms in breakthrough varicella cases, 
Table 3. Age-specific number of varicella cases by vaccination status and age-specific accumulative vaccination proportion of institutionalized population and during the 2008-2014 varicella outbreaks in Minhang District, Shanghai

\begin{tabular}{lllll}
\hline \hline $\begin{array}{l}\text { Age group } \\
\text { years })\end{array}$ & $\begin{array}{l}\text { Total cases } \\
(n=1558)\end{array}$ & $\begin{array}{l}\text { Number of vaccinated cases } \\
(\text { proportion } \%)(n=660)\end{array}$ & $\begin{array}{l}\text { Number of unvaccinated cases } \\
(\text { proportion \%) }(n=898)\end{array}$ & $\begin{array}{l}\text { Accumulative vaccination } \\
\text { proportion* }(\%)\end{array}$ \\
\hline$<1$ & 0 & $0(0)$ & $0(0)$ & 0 \\
$1-2$ & 0 & $0(0)$ & $0(0)$ & $77 \cdot 4$ \\
$3-4$ & 103 & $73(11 \cdot 1)$ & $30(3 \cdot 3)$ & $95 \cdot 8$ \\
$5-9$ & 770 & $435(65 \cdot 9)$ & $335(37 \cdot 3)$ & $89 \cdot 7$ \\
$10-14$ & 437 & $146(22 \cdot 1)$ & $291(32 \cdot 4)$ & $88 \cdot 8$ \\
$15-19$ & 207 & $6(0 \cdot 9)$ & $201(22 \cdot 4)$ & $54 \cdot 2$ \\
$20-24$ & 33 & $0(0)$ & $33(3 \cdot 7)$ & $23 \cdot 3$ \\
$\geqslant 25$ & 8 & $0(0)$ & $8(0 \cdot 9)$ & 0 \\
\hline \hline
\end{tabular}

* Data on accumulative vaccination proportion were estimated through Immunization Information Registry System since 2013.

Table 4. Breakthrough varicella infection in institution outbreaks in Minhang District during 2008-2014

\begin{tabular}{llllll}
\hline \hline Year & $\begin{array}{l}\text { Number of } \\
\text { outbreaks }\end{array}$ & $\begin{array}{l}\text { Number of } \\
\text { outbreak-associated cases }\end{array}$ & $\begin{array}{l}\text { Number of } \\
\text { breakthrough cases } \\
\text { (proportion) }\end{array}$ & $\begin{array}{l}\text { Number of vaccinated } \\
\text { children in outbreak } \\
\text { classes* }\end{array}$ & $\begin{array}{l}\text { Breakthrough infection } \\
\text { rate in outbreak classes* }\end{array}$ \\
\hline 2008 & 38 & 368 & $79(21 \cdot 5 \%)$ & $/$ & $/$ \\
2009 & 31 & 200 & $61(30 \cdot 5 \%)$ & $/$ & $/$ \\
2010 & 17 & 233 & $99(42 \cdot 5 \%)$ & $/$ & $/$ \\
2011 & 22 & 281 & $86(30 \cdot 6 \%)$ & $/$ & $/$ \\
2012 & 16 & 143 & $67(46 \cdot 9 \%)$ & $/$ & $7 \cdot 4 \%$ \\
2013 & 9 & 96 & $64(66 \cdot 7 \%)$ & 861 & $5 \cdot 4 \%$ \\
2014 & 21 & 237 & $204(86 \cdot 1 \%)$ & 3753 & $/$ \\
Total & 154 & 1558 & $660(42 \cdot 4 \%)$ & $/$ & $/$ \\
\hline \hline
\end{tabular}

' $l$ : data unavailable.

* Vaccination status in outbreak classes can be available since 2013 through Immunization Information Registry System and Immunization Records.

but also the longer average duration of outbreaks in vaccinated cases as index cases than in unvaccinated cases as index cases. The American Advisory Committee on Immunization Practices in 2006 recommended a second dose of varicella vaccine at $4-6$ years of age [14]. Since implementation of the second dose, studies have showed that varicella incidence has declined further. Effectiveness of the two-dose series was calculated to be $98 \%$ in a randomized, prospective study and a case-control study $[15,16]$. The breakthrough rate of two-dose vaccination was lower than that of one-dose vaccination $(2 \cdot 2 \%$ verse $7 \cdot 3 \%)$ after 10 years since vaccination [17]. The mathematical modeling suggested that the two-dose strategy can produce less natural varicella cases, but also considerably fewer breakthrough varicella cases [18]. Based on the epidemiologic studies from developed countries and our country, a routine two-dose varicella vaccination program should be implemented to improved vaccine-induced immunity.

Several outbreak investigations have suggested a longer time since vaccination as a risk factor for developing breakthrough varicella $[2,4,11,19-22]$. In this study, the mean time since vaccination was $6 \cdot 2$ years for breakthrough cases, and breakthrough varicella in institutions increased within 7 years after the first-dose vaccination. This result was consistent with the findings in other studies [4, 12, 22]. Galil et al. reported that children who had been vaccinated for more than 3 years were at twofold greater risk of varicella infection than those who were vaccinated more recently [21]. In Ozakai's study administration of a single dose of varicella vaccine could lead to the primary vaccine failure, as evidenced by non-seroconversion [23]. These results 
Table 5. Interval between breakthrough infection and vaccination

\begin{tabular}{lll}
\hline \hline $\begin{array}{l}\text { Time since } \\
\text { vaccination (years) }\end{array}$ & $\begin{array}{l}\text { Number of } \\
\text { breakthrough } \\
\text { cases }(n=660)\end{array}$ & Proportion (\%) \\
\hline$<1$ & 10 & $1 \cdot 5$ \\
1 & 8 & $1 \cdot 2$ \\
2 & 38 & $5 \cdot 8$ \\
3 & 86 & $13 \cdot 0$ \\
4 & 94 & $14 \cdot 2$ \\
5 & 89 & $13 \cdot 5$ \\
6 & 101 & $15 \cdot 3$ \\
7 & 108 & $16 \cdot 4$ \\
8 & 48 & $7 \cdot 3$ \\
9 & 37 & $5 \cdot 6$ \\
10 & 23 & $3 \cdot 5$ \\
11 & 12 & $1 \cdot 8$ \\
12 & 5 & $0 \cdot 8$ \\
13 & 1 & $0 \cdot 2$ \\
\hline \hline
\end{tabular}

illustrated that the immunity provided from varicella vaccines wanes over time and breakthrough infection may follow. Based on our study, it is optimal for children to receive a second booster dose of varicella vaccine 3 years after the first-dose vaccination. We also noticed the occurrence of $1.5 \%$ breakthrough infection within 1 year following the first-dose vaccination, which could be attributable to primary vaccine failure. Primary vaccine failure was observed in around $5 \%$ of vaccine recipients after administration of a single dose of varicella vaccine [23]. The data from Japan demonstrated that additional varicella vaccination after the initial vaccination could improve protection from both primary vaccine failure and waning vaccine-induced immunity [23]. Implementation of a two-dose varicella vaccine strategy has effectively mitigated outbreaks in school and kindergarten settings in Beijing since 2012 [24]. Additionally, we found no significant difference in average interval between vaccination and breakthrough infection for imported Varilrix ${ }^{\circledR}$ (GlaxoSmithKline) and domestic vaccine (Shanghai Institute of Biological Products). This finding further supports the similar effectiveness of onedose imported Varilrix ${ }^{\mathbb{B}}$ and domestic vaccine [13].

Our findings suggest that breakthrough varicella was common in the outbreak institutions and attributable primarily to waning vaccine-induced immunity over time. Currently, the wild VZV strains causing the varicella outbreaks in Minhang District of Shanghai were almost highly homologous to the vaccine Oka strain, and belonged to $\mathrm{J}$ genotype, indicating the match between the epidemic strain and the vaccine strain [25]. Special emphasis should be placed on varicella booster vaccination for children prior to school entry and catch-up vaccination for susceptible unvaccinated children and adolescent in order to prevent and control varicella outbreaks. Two-dose varicella vaccination should be included into the national childhood immunization program.

\section{ACKNOWLEDGEMENTS}

The authors sincerely thank the staff of Center for Disease Control and Prevention and the school teachers and nurses, who participated in outbreak investigation and immunization record review. They also appreciated the collaboration of parents during investigation. This study was funded by a grant from the Three-Year Action and Plan of Key Academic Discipline Project of Shanghai Public Health (15GWZK0101) supported by Shanghai Municipal Commission of Health and Family Planning.

\section{DECLARATION OF INTEREST}

None.

\section{REFERENCES}

1. Arvin AM. Varicella-zoster virus. In: Long SS, Pickering LK, Prober CG, eds. Principles and Practice of Pediatric Infectious Diseases. New York: Churchill Livingstone, 2008, pp. 1021-1029.

2. Huang WC, et al. Varicella breakthrough infection and vaccine effectiveness in Taiwan. Vaccine 2011; 29: 2756 2760.

3. Baxter R, et al. Long-term effectiveness of varicella vaccine: a 14-year, prospective cohort study. Pediatrics 2013; 131: e1389-e1396.

4. Lopez AS, et al. One dose of varicella vaccine does not prevent school outbreaks: is it time for a second dose? Pediatric 2006; 117: e1070-e1077.

5. Lu L, et al. A varicella outbreak in a school with high one-dose vaccination coverage, Beijing, China. Vaccine 2012; 30: 5094-5098.

6. Wang $\mathbf{Z}$, et al. Single-dose varicella vaccine effectiveness in school settings in China. Vaccine 2013; 31: 3834 3838 .

7. Hambleton S, Gershon AA. The impact of varicella vaccination in the United States. Seminars in Pediatric Infectious Diseases 2005; 16: 38-43.

8. Seward JF, Marin M, Vazquez M. Varicella vaccine effectiveness in the US vaccination program: a review. The Journal of Infectious Diseases 2008; 197(Suppl. 2): 82-89.

9. Davis MM, Patel MS, Gebremariam A. Decline in varicella-related hospitalizations and expenditures for 
children and adults after introduction of varicella vaccine in the United States. Pediatrics 2004; 114: 786-792.

10. Nguyen HQ, Jumaan AO, Seward JF. Decline in mortality due to varicella after implementation of varicella vaccination in the United States. The New England Journal of Medicine 2005; 352: 450-458.

11. Chaves SS, et al. Loss of vaccine-induced immunity to varicella over time. The New England Journal of Medicine 2007; 356: 1121-1129.

12. Zhang X, et al. The epidemiology of varicella cases among children in Beijing's Fengtai District from 2008 to 2012. Vaccine 2014; 32: 3569-3572.

13. Wu HY, et al. An epidemiological investigation on varicella vaccination among children in some areas of Shanghai. Shanghai Journal of Preventive Medicine 2006; 18: 437-439.

14. Marin M, et al. Prevention of varicella: recommendations of the Advisory Committee on Immunization Practices (ACIP). Morbidity and Mortality Weekly Report Recommendations and Reports 2007; 56: 1-40.

15. Kattan JA, et al. Impact of 2-dose vaccination on varicella epidemiology: Connecticut-2005-2008. The Journal of Infectious Diseases 2011; 203: 509-512.

16. Shapiro ED, et al. Effectiveness of 2 doses of varicella vaccine in children. The Journal of Infectious Diseases 2011; 203: 312-315.

17. Kuter B, et al. Ten year follow-up of healthy children who received one or two injections of varicella vaccine. The Pediatric Infectious Disease Journal 2004; 23: 132-137.
18. Gao Z, et al. Modelling the impact of one-dose vs. twodose vaccination regimens on the epidemiology of varicella zoster virus in Australia. Epidemiology and Infection 2010; 138: 457-468.

19. Tugwell BD, et al. Chickenpox outbreak in a highly vaccinated school population. Pediatrics 2004; 113: 455-459.

20. Haddad MB, et al. Vaccine effectiveness during a varicella outbreak among schoolchildren: Utah, 20022003. Pediatrics 2005; 115: 1488-1493.

21. Galil K, et al. Outbreak of varicella at a day-care center despite vaccination. The New England Journal of Medicine 2002; 347: 1909-1915.

22. Kurugol Z, et al. Varicella rates among unvaccinated and one-dose vaccinated healthy children in Izmir, Turkey. International Journal of Infectious Diseases 2011; 15: e475-e480.

23. Ozaki T. Long-term clinical studies of varicella vaccine at a regional hospital in Japan and proposal for a varicella vaccination program. Vaccine 2013; 31: 6155-6160.

24. Suo L, et al. Effect evaluation of a 2 dose varicella vaccine immunization strategy implemented to control outbreaks in school and kindergarten settings. Chinese Journal of Preventive Medicine 2015; 49: 485-489.

25. Lu J, et al. Genetic characteristics of breakthrough varicella-zoster virus infection during the varicella outbreaks in Minhang District of Shanghai. Chinese Journal of Microbiology and Immunology 2016; 36: 271-276. 